\title{
EDITORIAL
}

\section{Silent gastro-oesophageal reflux and microaspiration in IPF: mounting evidence for anti-reflux therapy?}

\author{
G. Raghu and K.C. Meyer
}

I diopathic pulmonary fibrosis (IPF) is a distinct clinical entity that generally affects people who are over $60 \mathrm{yrs}$ of age, and median survival ranges from 3 to 5 yrs after the diagnosis is ascertained. The natural course of IPF is well known: the majority of patients with IPF display steady decline in lung function, some patients remain stable for prolonged periods of time, a subgroup of patients rapidly decline, and a subset of patients manifest acute exacerbations of IPF preceding death [1]. While several key cellular and molecular events that are thought to follow "injury" have been identified in the pathogenesis of IPF [2], the ultimate cause of IPF and the triggering factor(s) that injure the lung remain elusive, and none of the currently available pharmacological agents have demonstrated improved outcomes and survival in patients with IPF.

The incidence and prevalence of IPF is highly linked to advanced age with an estimated incidence and prevalence of 71 and 271 per 100,000 per year for males and 67 and 266 per 100,000 per year for females aged 75 yrs or greater versus an overall incidence and prevalence of 16.3 and 42.7 per 100,000 per year using broad diagnostic criteria [3]. Interestingly, many observations suggest that the aged lung is more susceptible to injury and fibrosis induced by a variety of stimuli, and this susceptibility may be linked to ageassociated changes in gene expression or genetic polymorphisms such as age-associated telomerase dysfunction [4-7].

Advanced age is also accompanied by a decline in oesophageal and gastric motility, diminished upper oesophageal sphincter pressure, and an increase in oesophageal acid exposure [8]. Hiatal hernias appear with advancing age and have been detected in up to $60 \%$ of individuals older than $60 \mathrm{yrs}$ [9]. The formation of a hiatal hernia, which tends to increase in size with advancing age, is associated with lower oesophageal sphincter dysfunction (diminished lower oesophageal sphincter basal pressure, greater oesophageal acid exposure and increased likelihood of erosive oesophagitis) [9]. The association of the presence of hiatal hernia and/or gastro-oesophageal reflux (GER) with pulmonary fibrosis has been well documented [10-12]. The severity and frequency of symptoms associated with GER (heartburn, epigastric discomfort and regurgitation) tend to decrease in older individuals [13] and hence contributes to the concept of "silent/occult GER and microaspiration". When one considers the strong association of

Dept of Medicine, Divison of Pulmonary and Critical Care Medicine, University of Washington, Seattle, WA, USA

CORRESPONDENCE: G. Raghu, Dept of Medicine, Division of Pulmonary Critical Care Medicine, Box 356175, University of Washington, Seattle, WA 98195, USA. E-mail: graghu@uw.edu
GER, GER disease, hiatal hernia and IPF with advanced age, the possibility that GER and microaspiration are linked to the pathogenesis of IPF and/or episodes of acute exacerbations of IPF must be seriously considered.

Proximal GER has been documented in healthy subjects during sleep [14], and resting upper oesophageal sphincter pressure also falls considerably during sleep [15]. Indeed, GLEESON et al. [14] found that nearly half of the healthy adult subjects that they evaluated aspirated small amounts of their oropharyngeal secretions during sleep, indicating that "silent" microaspiration is a common phenomenon in adults. Microaspiration has been postulated as an aetiological factor in the pathogenesis of IPF, a hypothesis that is based on the abnormal, "silent" acid GER observed in $\sim 90 \%$ of patients with IPF based on 24-h oesophageal $\mathrm{pH}$ monitoring $[16,17]$. However, the lack of histological features of "micro and/or macro aspiration" in areas of usual interstitial pneumonia (UIP) raises an appropriate hesitation to the acceptance of a role for GER and microaspiration in the pathogenesis of IPF. Nonetheless, an increasing body of evidence that has accumulated over the past few years support the concept that occult microaspiration of refluxed gastric juice may play a significant role in the pathogenesis of IPF. Interestingly, these studies have shown that typical GER symptoms (heartburn and regurgitation) are poor predictors of GER in patients with IPF.

Despite the increasing number of studies that have documented a high prevalence of GER in patients with IPF [16-20], a causal relationship between GER and IPF has yet to be firmly established. Regardless, it is notable that a number of case series have suggest a link between GER suppression and clinical stabilisation. This has led to the clinical evaluation of "antireflux" therapy as the sole treatment for IPF in patients with abnormal GER. RAGHU et al. [21] described four patients with well-defined IPF whose clinical course stabilised or improved over a 4-yr period of time with the sole intervention of using antireflux therapy - the suppression of acid GER by proton pump inhibitors (PPIs) and/or anti-reflux surgery - was confirmed via 24-h oesophageal $\mathrm{pH}$ monitoring in all four patients. LINDEN et al. [22] reported a series of 14 patients and found that oxygen requirements stabilised in those patients who underwent Nissen fundoplication. More recently, LEE et al. [23] reported that use of agents to suppress GER in a cohort of 204 patients with IPF was associated with a lower radiological fibrosis score on highresolution computed tomography and was an independent predictor of longer survival time. The retrospective observation of enhanced survival in patients with IPF who were allegedly taking medications to suppress acidity of gastric secretions [23] 
implies that treatment with PPIs and/or $\mathrm{H} 2$ receptor antagonists may decrease the "injury" and/or prevent subsequent consequences of recurrent "injury" to the epithelial barrier of distal pulmonary parenchyma that may have been otherwise induced by intermittent episodes of microaspiration of the acid component of gastric juice.

An evolving approach to detecting reflux and associated microaspiration is the detection of biomarkers of aspiration in respiratory secretions such as pepsin. Bile acids and pepsin have been quantified in sputum and bronchoalveolar lavage (BAL) fluid as markers of microaspiration [24-27]. STAROSTA et al. [28] reported that the number of proximal reflux events detected by 24-h $\mathrm{pH}$ monitoring correlated with pepsin levels in BAL fluid. Although BAL pepsin or bile salt detection represents a direct marker of aspiration of refluxed gastric juice, further studies that measure pepsin and bile salts in BAL fluid are warranted to correlate with disease severity, risk of progression and/or the development of acute exacerbation in patients with IPF.

In this issue of the European Respiratory Journal, two independent groups of investigators have further explored the concept of microaspiration in IPF [29, 30]. Using multi-detector computerised tomographic imaging in patients with IPF, NoTH et al. [29] confirmed the previously known increased prevalence of hiatal hernia in patients with IPF, demonstrated that IPF patients with hiatal hernia had greater physiological impairment on pulmonary function testing than patients who did not have hiatal hernia, and, in a small subset of IPF patients who were subjected to 24-h $\mathrm{pH}$ monitoring $(\mathrm{n}=14)$, demonstrated that abnormal acid GER was associated with the presence of hiatal hernia. In a larger subset of 74 patients for whom data on the use of anti-reflux medications were available, the group receiving therapy had significantly better diffusing capacity of the lung for carbon monoxide and composite physiological index scores.

LEE et al. [30] are the first to have detected a biomarker of microaspiration, pepsin, in BAL fluid from patients with IPF. A substantial number of their patients had clinical evidence of acute exacerbations of IPF, and BAL pepsin and neutrophil percentages on differential BAL cell counts were significantly increased for the group with acute exacerbations of IPF versus stable patients. An increase in BAL pepsin level by one standard deviation from that of the stable group was associated with an odds ratio of $1.46(95 \%$ CI 1.03-2.09; $\mathrm{p}=0.04)$ for having an acute exacerbation. However, the prevalence or size of radiologically identified hiatal hernia did not correlate with exacerbation status. Additionally, BAL pepsin levels were not predictive of survival, and the increased pepsin levels were driven by a subgroup ( $33 \%$ of cases) with markedly elevated pepsin levels in BAL. Nonetheless, the observations in the retrospective study by LEE et al. [30] are intriguing, as the detection of pepsin in BAL fluid at the onset of an acute exacerbation of IPF episode is direct evidence that the contents of gastric juice can reach the lower respiratory tract without an overt aspiration event. These findings support the long-standing hypothesis that microaspiration plays a role in the pathogenesis of IPF and that the unknown "trigger" and/or the aetiological agent causing lung injury may be translocation of refluxed gastric juice to distal areas of the pulmonary parenchyma (fig. 1).

The clinical consequences of an aspiration event depend upon the nature (acidity, presence of pepsin, bile acids and other constituents of gastric juice), volume, frequency of aspiration, and the subject's ability to neutralise and clear the aspirated
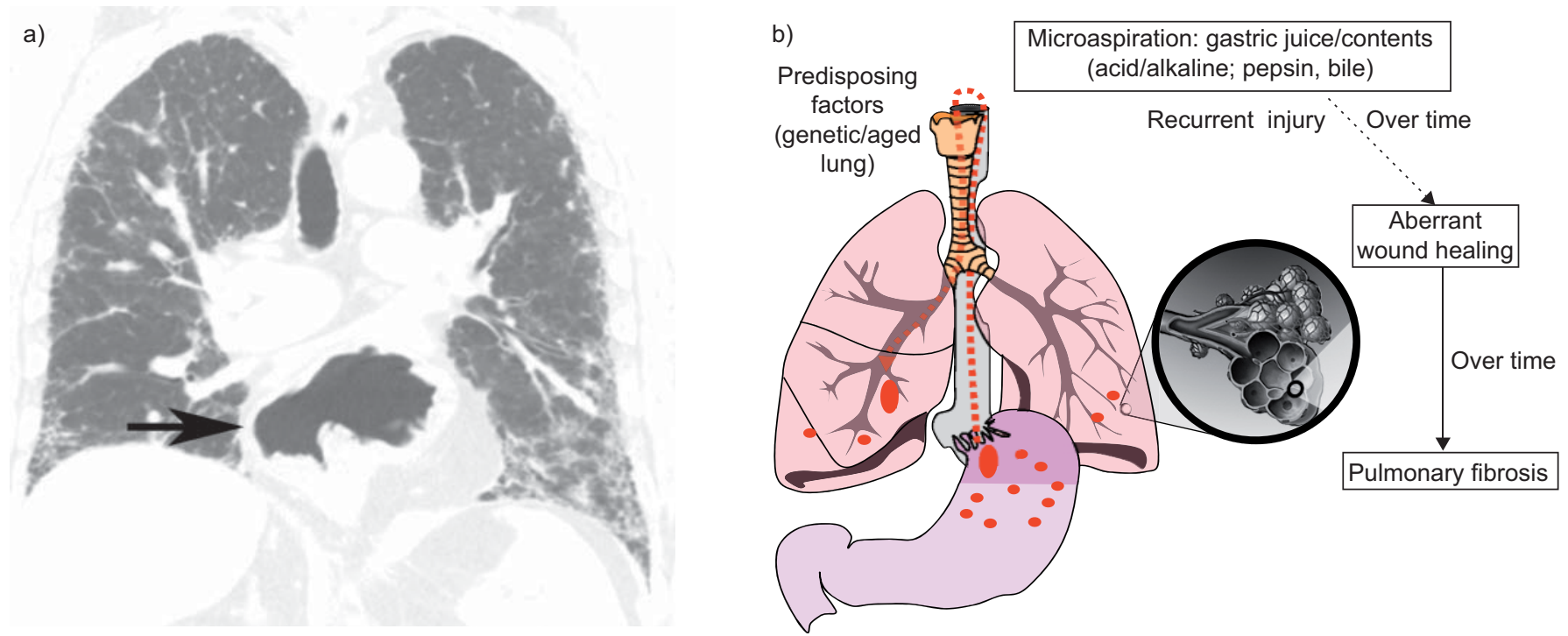

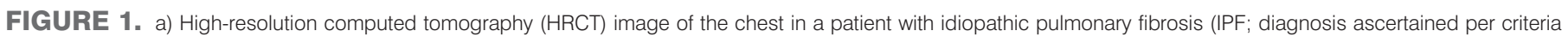

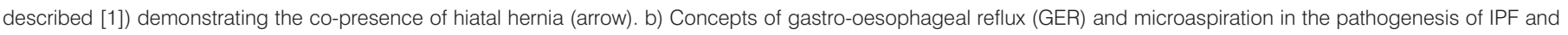

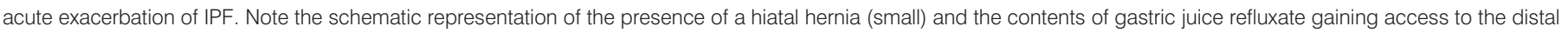

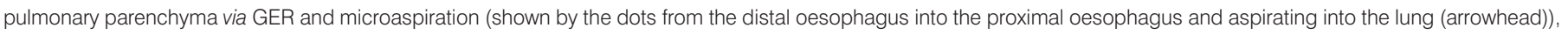

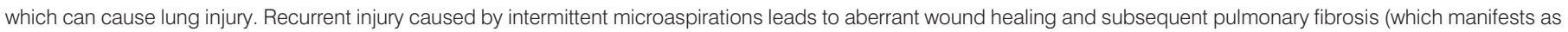

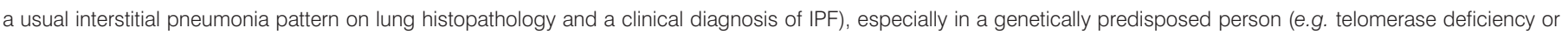

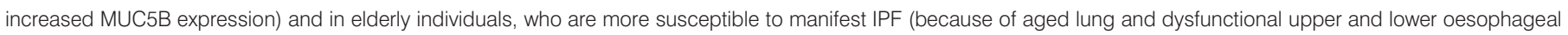
sphincters). 
material from the lungs and thereby prevent/limit mucosal damage, inflammation and subsequent pulmonary fibrosis. The observed association of hiatal hernia and abnormal acid GER coupled with the detection of pepsin in BAL retrieved from distal pulmonary parenchyma support the long-standing hypothesis that microaspiration is a causative role in IPF and may trigger episodes of acute exacerbations of IPF. These two reports further advance our knowledge of GER and microaspiration in the pathogenesis of IPF and support the concept that GER and microaspiration are associated with more severe pulmonary impairment in patients without acute exacerbations of IPF and that GER and occult aspiration may trigger an episode of acute respiratory decompensation. Microaspiration of gastric juice as a trigger of acute exacerbations is an attractive hypothesis, particularly when one considers the observations that the predominant histopathologic lesion of acute exacerbations of IPF is diffuse alveolar damage, which has been observed when acid or gastric juice is instilled into the lungs of laboratory animals, and that the cause of acute exacerbations of IPF is unknown. While acid is known to cause lung epithelial injury, the observation that non-acid components of gastric juice, such as pepsin, can be found in distal airways, especially during episodes of acute exacerbations of IPF cannot be ignored and has implications for therapeutic intervention. Indeed, pepsin in BAL fluid may well be considered a biomarker of microaspiration and may be useful to assess the efficacy of anti-reflux therapy. Pepsin or bile salts could be monitored in BAL fluid to determine the efficacy of anti-reflux surgical interventions such as fundoplication, as has been reported in lung transplant recipients with lung allograft dysfunction [31].

We speculate that the collision of advanced age and its associated changes in susceptibility to lung injury (which may be related to gene expression changes such as mutations in telomerase and MUC5B expression [32]) with an increased risk of having episodes of GER, hiatal hernia and microaspiration of gastric juice and its constituents, including the possibility of Helicobacter pylori, that may lead to lung epithelial injury, aberrant repair responses and fibrosis in susceptible individuals (fig. 1). Additionally, episodes of gastric juice microaspiration may lead to ongoing injury and fibrotic responses that lead to accelerated loss of lung function due to fibrotic responses that are characteristic of the UIP histopathology in IPF. Finally, episodes in which a larger bolus of gastric juice reaches the lung may trigger episodes of acute exacerbations of IPF.

The only intervention that has a significant impact on survival and quality of life in patients with IPF to date is lung transplantation, but only a minority of patients with IPF are acceptable candidates for this intervention [33]. No pharmacological treatment interventions evaluated in clinical trials have demonstrated a survival benefit for patients with IPF, and new approaches that can stabilise the disease and improve survival are sorely needed. The observations by NOTH et al. [29] and LEE et al. [30] clearly warrant further investigation and have implications for anti-reflux measures, including conservative measures (avoiding total recumbence, limited meal size, avoiding certain foods, avoiding alcohol or caffeinated beverages, etc.) to decrease GER and/or laparoscopic anti-reflux surgery (e.g. Nissen fundoplication) that may be important therapeutic interventions, rather than merely suppressing the acidity of refluxed gastric secretions by treating patients with acid-suppressing agents such as PPIs and $\mathrm{H} 2$ receptor antagonists.

Additional research on the role of GER and microaspiration in the pathogenesis of IPF should be pursued to gain further insights that lead to a better understanding of pathogenesis and to determine the role of anti-reflux therapies in the stabilisation of IPF and prevention of acute exacerbations. Although the use of pharmacological agents that suppress acidity have been associated with disease stabilisation and improved survival, it must be emphasised that the GER and aspiration per se are not suppressed by PPI, and aspiration with alkaline gastric juice can still occur. Strategies to use impedance $\mathrm{pH}$ monitoring and to detect biomarkers of microaspiration need to be established in order to allow reliable screening and monitoring for significant GER and microaspiration, and to correlate such findings with the disease course in patients with IPF. It is hoped that treatment strategies to prevent GER will protect the already injured lung from further insults and preserve the microenvironment of gas exchange units of the lung, thus leading to improved outcomes for patients with IPF.

\section{STATEMENT OF INTEREST}

None declared.

\section{REFERENCES}

1 Raghu G, Collard HR, Egan JJ, et al. An official ATS/ERS/JRS/ ALAT statement: idiopathic pulmonary fibrosis: evidence-based guidelines for diagnosis and management. Am J Respir Crit Care Med 2011; 183: 788-824.

2 Raghu G, Narayanan A, Khalil N. Pathogenesis of Idiopathic Pulmonary Fibrosis. www.uptodate.com/contents/pathogenesisof-idiopathic-pulmonary-fibrosis Date last updated: June 7, 2011.

3 Raghu G, Weycker D, Edelsberg J, et al. Incidence and prevalence of idiopathic pulmonary fibrosis. Am J Respir Crit Care Med 2006; 174: 810-816.

4 King TE Jr, Pardo A, Selman M. Idiopathic pulmonary fibrosis. Lancet 2011; 378: 1949-1961.

5 Collins K, Mitchell JR. Telomerase in the human organism. Oncogene 2002; 21: 564-579.

6 Liu T, Hu B, Chung MJ, et al. Telomerase regulation of myofibroblast differentiation. Am J Respir Cell Mol Biol 2006; 34: 625-633.

7 Tsakiri K, Cronkhite JT, Kuan PJ, et al. Adult-onset pulmonary fibrosis caused by mutations in telomerase. Proc Natl Acad Sci USA 2007; 104: 7552-7557.

8 Poh $\mathrm{CH}$, Navarro-Rodrigues T, Fass R. Review: treatment of gastroesophageal reflux disease in the elderly. Am J Med 2010; 123: 496-501.

9 Patti MG, Goldberg HI, Arcerito M, et al. Hiatal hernia size affects lower esophageal sphincter function, esophageal acid exposure, and the degree of mucosal injury. Am J Surg 1996; 171: 182-186.

10 Pearson JE, Wilson RS. Diffuse pulmonary fibrosis and hiatus hernia. Thorax 1971; 26: 300-305.

11 Mays EE, Dubois JJ, Hamilton GB. Pulmonary fibrosis associated with tracheobronchial aspiration. A study of the frequency of hiatal hernia and gastroesophageal reflux in interstitial pulmonary fibrosis of obscure etiology. Chest 1976; 69: 512-515.

12 el-Serag HB, Sonnenberg A. Comorbid occurrence of laryngeal or pulmonary disease with esophagitis in United States military veterans. Gastroenterology 1997; 113: 755-760.

13 Pilotto A, Franceschi M, Leandro G, et al. Clinical features of reflux esophagitis in older people: a study of 840 consecutive patients. J Am Geriatr Soc 2006; 54: 1537-1542. 
14 Gleeson K, Eggli DF, Maxwell SL. Quantitative aspiration during sleep in normal subjects. Chest 1997; 111: 1266-1272.

15 Kahrilas PJ, Dodds WJ, Dent J, et al. Effect of sleep, spontaneous gastroesophageal reflux, and a meal on upper esophageal sphincter pressure in normal human volunteers. Gastroenterology 1987; 92: 466-471.

16 Tobin RW, Pope CE II, Pellegrini CA, et al. Increased prevalence of gastroesophageal reflux in patients with idiopathic pulmonary fibrosis. Am J Respir Crit Care Med 1998; 158: 1804-1808.

17 Raghu G, Freudenberger TD, Yang S, et al. High prevalence of abnormal acid reflux in idiopathic pulmonary fibrosis. Eur Respir J 2006; 27: 136-142.

18 Patti MG, Tedesco P, Golden J, et al. Idiopathic pulmonary fibrosis: how often is it really idiopathic? J Gastrointest Surg 2005; 9: 1053-1056.

19 Salvioli B, Belmonte G, Stanghellini V, et al. Gastro-oesophageal reflux and interstitial lung disease. Dig Liver Dis 2006; 38: 879-884.

20 Sweet MP, Patti MG, Leard LE, et al. Gastroesophageal reflux in patients with idiopathic pulmonary fibrosis referred for lung transplantataion. J Thorac Cardiovasc Surg 2007; 133: 1078-1084.

21 Raghu G, Yang ST, Spada C, et al. Sole treatment of acid gastroesophageal reflux in idiopathic pulmonary fibrosis: a case series. Chest 2006; 129: 794-800.

22 Linden PA, Gilbert RJ, Yeap BY, et al. Laparoscopic fundoplication in patients with end-stage lung disease awaiting transplantation. J Thorac Cardiovasc Surg 2006; 131: 438-446.

23 Lee JS, Ryu JH, Elicker BM, et al. Gastroesophageal reflux therapy is associated with longer survival in idiopathic pulmonary fibrosis. Am J Respir Crit Care Med 2011 [Epub ahead of print DOI: $10.1164 / \mathrm{rccm} .201101-0138 \mathrm{OC}]$
24 Metheny NA, Chang YH, Ye JS, et al. Pepsin as a marker for pulmonary aspiration. Am J Crit Care 2002; 11: 150-154.

25 Potluri S, Friedenberg F, Parkman HP, et al. Comparison of a salivary/sputum pepsin assay with 24-hour esophageal $\mathrm{pH}$ monitoring for detection of gastric reflux into the proximal esophagus, oropharynx, and lung. Dig Dis Sci 2003; 48: 1813-1817.

26 D'Ovidio F, Mura M, Waddell TK, et al. Bile acids in bronchoalveolar lavage after lung transplantation as a marker of pulmonary aspiration associated with alveolar neutrophilia. J Heart Lung Transplant 2004; 23: Suppl., 42S.

27 Stovold R, Forrest IA, Corris PA, et al. Pepsin, a biomarker of gastric aspiration in lung allografts: a putative association with rejection. Am J Respir Crit Care Med 2007; 175: 1298-1303.

28 Starosta V, Kitz R, Hartl D, et al. Bronchoalveolar pepsin, bile acids, oxidation, and inflammation in children with gastroesophageal reflux disease. Chest 2007; 132: 1557-1564.

29 Noth I, Zangan SM, Soares RV, et al. Prevalence of hiatal hernia by blinded multidetector CT in patients with idiopathic pulmonary fibrosis. Eur Respir J 2012; 39: 344-351.

30 Lee JS, Song JW, Wolters PJ, et al. Bronchoalveolar lavage pepsin in acute exacerbation of idiopathic pulmonary fibrosis. Eur Respir $J$ 2012; 39: 352-358.

31 Fisichella PM, David CS, Lundberg PW, et al. The protective role of laparoscopic antireflux surgery against aspiration of pepsin after lung transplantation. Surgery 2011; 150: 598-606.

32 Seibold MA, Wise AL, Speer MC, et al. A common MUC5B promoter polymorphism and pulmonary fibrosis. $N$ Engl J Med 2011; 364: 1503-1512.

33 Nathan SD, Shlobin OA, Weir N, et al. Long-term course and prognosis of idiopathic pulmonary fibrosis in the new millennium. Chest 2011; 140: 221-229. 